\title{
Unified Study of Electrical Resistivity of Simple and Non-Simple Liquid Metals
}

\author{
J. K. Baria \\ V. P. \& R. P. T. P. Science College, Vallabh Vidyanagar-388 120, Gujarat, India
}

Received on 29 October, 2003; revised version received on 4 January, 2004

\begin{abstract}
A model potential depending on an effective core radius but otherwise parameter free is used for the comparative study of electrical resistivity of simple and non-simple liquid metals. In the present paper electrical resistivity of simple and non-simple liquid metals have been calculated using Ziman's formula, Ziman's formula modified and used by Khajil and Tomak (Self consistent approximation) and t-matrix formulation given by Evans and Evans et al. Previously no one have reported such comparative study using pseudopotentials. In the electrical resistivity calculations we have used structure factor derived through charge hard sphere approximation. The beauty of this approximation is that it needs pseudopotential form factor for the calculation of structure factor. So this gives the better explanation of structure factor than any other approximations. From present investigations it is found that self consistent formulation results are excellently agrees with the experimental findings. A successful application is an evidence that our potential can predict wide class of physical properties of $\mathrm{d}$ and $\mathrm{f}$ shell metals as well as simple and non-simple metals.
\end{abstract}

\section{Introduction}

The Ziman's nearly free electron (NFE) theory has been fairly successful in describing the quantitative behavior of the electrical resistivity in simple liquid metals. This is because in these metals the mean free path is about one hundred times the interatomic distance and the weak scattering picture should be valid. Even for the heavy polyvalent metals (e.g. mercury, thallium and lead) where the mean free path is only about two interatomic distances, the NFE model can yield results, which are in reasonable in agreement with experiments. Calculations of electrical Resistivity using structure factor from various experiments or different versions of bare ion potential and dielectric function, gives correct order of magnitude but differ among themselves. In fact sometimes the theory is trusted sufficiently to use measured value of Resistivity to determine the parameter of the potential. The Ziman's formula is not expected to apply to d-state transition metals because the unfilled d-state cause strong resonant scattering which seems inappropriate for description by pseudopotential. Nevertheless, Evans et al [1] put forward a version of equation of Resistivity in which $|W(q)|^{2}$ was replaced by exact value of the squared matrix element for the scattering of a plane wave by a transition metal ion. The potential of the latter was taken to be MuffinTin potential as derived in solid state physics for band structure calculations and the exact scattering can be calculated by the phase shift method.

\section{Electrical resistivity of liquid met- als}

For both liquid and amorphous metals the electrical resistivity is given by $[1,2]$

$$
\rho_{\text {Ziman }}=\frac{3 \pi m^{2}}{4 Z e^{2} \hbar^{3} n k_{F}^{6}} \int_{0}^{\infty} q^{3} S(q)|W(q)|^{2} \Theta\left(2 k_{F}-q\right) d q
$$

Here, $\mathrm{S}(\mathrm{q})$ is the structure factor. In the present work we have used the Charged Hard Sphere (CHS) [3] structure factor. The structure factor derived through CHS has an advantage that it involves the pseudopotential form factor. In this sense it differs from the Percus-Yevick (PY) [4] theory.
So the structure factor derived through CHS gives the better explanation than PY theory. Recently Baria and Jani $[5,6]$ have proposed model potential which is quite successful in describing lattice mechanical properties of $\mathrm{d}$ and $\mathrm{f}-$ shell metals is used in the present calculation of electrical resis- 
tivity for simple and non-simple liquid metals. The unit step function $\Theta$ which cuts off the integration at $2 \mathrm{k}_{F}$ corresponding to a preferably sharp Fermi surface, is defined as,

$$
\begin{aligned}
\Theta\left(2 k_{F}-q\right) & =0 \text { for } q>2 k_{F} \\
& =1 \text { for } q \leq 2 k_{F}
\end{aligned}
$$

But the finite mean free path corresponds to a finite uncertainty in the electron position. This corresponds to a finite uncertainty in the electron momentum. Thus the Fermi surface is not perfectly sharp as implied by the equation (1) but it is blurred. The attempts to take this blurring in to account in the formulation of resistivity is reviewed by Mc Caskill and March [7]. Ferraz and March [8] approach yields in place of equation (1)

$$
\rho_{s c}=\frac{3 \pi m^{2}}{4 Z e^{2} \hbar^{3} n k_{F}^{6}} \int_{0}^{\infty} q^{4} S(q)|W(q)|^{2} \Gamma\left(q, k_{F}, l\right) d q
$$

This equation must self consistently solved. Very few explicit approximations are proposed for the function $\Gamma\left(q, k_{F}, l\right)[7]$

In this work we have used the form for $\Gamma\left(q, k_{F}, l\right)$ as used by Laakkonen and Nieminen [9] and Khajil and Tomak [10].

$$
\Gamma\left(q, k_{F}, l\right)=\frac{2}{\pi q^{3}}\left[\tan ^{-1}(q l)-\frac{1}{2} \tan ^{-1}\left(\frac{2 q l}{1+4\left(k_{F} l\right)^{2}-(q l)^{2}}\right)-\frac{\pi}{2} \Theta\left(q-\left(\frac{1}{l^{2}}+4 k_{F}^{2}\right)\right)\right]
$$

The mean free path is determined self consistently as follows. The first step of the self-consistency loop is to calculate resistivity using equation (1) i.e. with mean free path " $l$ " is infinity. A new mean free path " $l$ " is then obtained from the Drude relation as

$$
\rho_{L}=\frac{\hbar k_{F}}{n e^{2} l}
$$

The iterations are continued till $\rho_{L}$ converges.

\section{Resistivity (t-matrix) approach}

We are concerned in this section with the scattering occurring in liquid metals. For example, a flux of electrons is incident on a scattering center and it is important to know the number of electrons that are scattered in a definite direction. This problem is most conveniently discussed using phase analysis. This analysis will throw much additional light on the use of pseudopotential see for more detail [11-12]. For many purposes a detailed knowledge of how the electronic wave functions behave in the neighborhood of the perturbing potential is not required, it is only their asymptotic form that matters. This, of course, is completely determines by phase shifts. Since, when the potential is due to something as small as metallic ion, the phase shifts are normally insignificant beyond say $\eta_{3}$ therefore $\eta_{0}, \eta_{1}$ and $\eta_{2}$ are to be calculated.

The phase-shift $\eta_{l}$ of the $l^{t h}$ partial wave related to the pseudopotential is given by

$$
\eta_{l}=-\frac{m k_{F} \Omega_{0}}{4 \pi \hbar^{2}} \int_{0}^{2} W(y) \text { y } P_{l}(\cos \theta) d y
$$

where $\mathrm{y}=\mathrm{q} / 2 \mathrm{kF}$ and $\mathrm{P}_{l}(\cos \theta)$ is a Legendre polynomial and $\cos \theta=1-\frac{1}{2}\left(\frac{q}{k_{F}}\right)^{2}$

Using the above equation phase-shift $\eta_{0}, \eta_{1}, \eta_{2}$ are calculated for $\mathrm{s}, \mathrm{p}$ and $\mathrm{d}$ components and are shown in the table I. Note that the low-energy phase-shift obeys the rule $\eta_{0}>\eta_{1}>\eta_{2}$. The larger the phase-shift, the stronger the scattering. Evidently it is liable to be especially strong if the energy of the incident electron happens to coincide with that of a virtual bound state.

TABLE I. Presently calculated values of phase shift of some simple and non-simple liquid metals.

\begin{tabular}{|l|l|l|l|}
\hline Metal & $\eta_{0}$ & $\eta_{1}$ & $\eta_{2}$ \\
\hline $\mathrm{Li}$ & 0.374 & 0.302 & 0.053 \\
\hline $\mathrm{Na}$ & 0.674 & 0.265 & 0.023 \\
\hline $\mathrm{K}$ & 0.515 & 0.322 & 0.025 \\
\hline $\mathrm{Rb}$ & 0.782 & 0.265 & 0.003 \\
\hline $\mathrm{Cs}$ & 0.781 & 0.272 & 0.001 \\
\hline $\mathrm{Be}$ & 0.757 & 0.491 & 0.129 \\
\hline $\mathrm{Mg}$ & 0.882 & 0.544 & 0.105 \\
\hline $\mathrm{Ca}$ & 0.445 & 0.252 & 0.013 \\
\hline $\mathrm{Sr}$ & 0.795 & 0.619 & 0.095 \\
\hline $\mathrm{Ba}$ & 0.758 & 0.634 & 0.095 \\
\hline $\mathrm{Zn}$ & 1.148 & 0.469 & 0.092 \\
\hline $\mathrm{Cd}$ & 1.1874 & 0.486 & 0.083 \\
\hline $\mathrm{Hg}$ & 1.302 & 0.458 & 0.069 \\
\hline $\mathrm{Al}$ & 1.074 & 0.777 & 0.195 \\
\hline $\mathrm{Ga}$ & 1.392 & 0.737 & 0.167 \\
\hline $\mathrm{In}$ & 1.381 & 0.771 & 0.162 \\
\hline $\mathrm{Pb}$ & 1.889 & 1.007 & 0.215 \\
\hline
\end{tabular}

In the case of noble and transition metals, the NFE approach is not appropriate due to presence the presence of a $\mathrm{d}$ band in the conduction band, which complicates the picture. We use the t-matrix of the pseudopotential to calculate 
the scattering cross section rather than the usual screened ion model potentials. The single scatter t-matrix form factor for energy conserving transition is given by Evans [13] and Evans et al [14]

$$
t\left(\vec{k}, \vec{k}^{\prime}\right)=-\frac{2 \pi \hbar^{3}}{m(2 m E)^{\frac{1}{2}}} \frac{1}{\Omega_{0}} \sum_{l}(2 l+1) \sin \eta_{l}\left(E_{F}\right) \exp \left[i \eta_{l}\left(E_{F}\right)\right] P_{l}(\cos \theta)
$$

The t-matrix has the dimensions of energy and is normalized to the atomic volume. The resistivity takes the form with $\mathrm{y}=\mathrm{q} / 2 \mathrm{k}_{F}$

$$
\rho_{t}=\frac{3 \pi \Omega_{0}}{e^{2} \hbar^{2} v_{F}^{2}} \int_{0}^{1} 4 y^{3} S(q)\left|t\left(\vec{k}, \vec{k}^{\prime}\right)\right|^{2} d y
$$

\section{$4 \quad$ Results and discussion}

The resistivity calculated using Ziman's formula, selfconsistent approach and t-matrix formulations are tabulated in table II with the experimental and other such theoretical findings. Daver et al [15] have calculated resistivity and the mean free path using self-consistent method of about twenty liquid metals inclusive noble metals. They have used the Ashcroft's empty-core pseudopotential, the parameter of the potential were chosen to yield the best agreement with the experimental structure factor. As they have fitted the potential parameter their results are very close to the experi- mental findings for simple metals while for $\mathrm{Cu}$ and $\mathrm{Ag}$ it deviates little. Very recently Geertsma et al [16] have calculated density of states, resistivity and thermopower of liquid alkali metals and two liquid alloys Li-Na and $\mathrm{Na}-\mathrm{K}$. they have used Linear Response Theory (LRT) to calculate the screened pseudopotential and the structure factor was obtained from the pair-potential using Modified Hyper Neted Chain (MHNC) theory of liquid. Their results of electrical resistivity for liquid alkali metals are very poor particularly for $\mathrm{Li}$ and Cs. Leavens et al [17] have also calculated electrical resistivity using Ziman's formula and self consistent approximation for liquid alkali metals $\mathrm{Mg}$ and $\mathrm{Al}$. their results of electrical resistivity using self consistent approximation are better than Ziman's formula but could not avoid the reasonable deviation from the experimental findings. It is evident from table 2 that the electrical resistivity using self consistent approach is much better than the Zimzn's formula except $\mathrm{Al}$, Ga and In, while t-matrix results are deviating much from the experimental findings. Moreover the mean free path of the present investigations is also comparable to the previously reported theoretical findings $[15,17]$.

\begin{tabular}{|c|c|c|c|c|c|c|c|c|c|c|c|c|}
\hline \multirow{2}{*}{ Metal } & \multicolumn{3}{|l|}{$l$ in $(\mathrm{au})$} & \multicolumn{3}{|c|}{ Electrical resistivity $(\rho)$} & \multirow{2}{*}{$\rho_{\text {Expt }} \cdot[11$} & \multicolumn{5}{|c|}{ Other theoretical findings } \\
\hline & Present & $\begin{array}{l}\text { Others } \\
{[15]}\end{array}$ & $\begin{array}{l}\text { Others } \\
{[17]}\end{array}$ & $\rho_{s c}$ & $\rho_{\text {Ziman }}$ & $\rho_{T-m a t}$ & & [15] & [16] & [16] & [18] & [11] \\
\hline $\mathrm{Li}$ & 108.48 & 77.5 & 112.05 & 23.8 & 21.15 & 18.91 & 24.7 & 23.2 & 7.0 & 7.3 & & 25 \\
\hline $\mathrm{Na}$ & 192.29 & 288.0 & 165.91 & 9.82 & 8.44 & 37.7 & 9.6 & 5.3 & 15.8 & 16.3 & & 7.9 \\
\hline $\mathrm{K}$ & 269.09 & 316.0 & 182.41 & 14.06 & 11.48 & 26.11 & 13.0 & 11.1 & 18.5 & 19.6 & & 23 \\
\hline $\mathrm{Rb}$ & 174.11 & 223.0 & 159.76 & 23.09 & 23.62 & 30.66 & 22.5 & 19.8 & 20.8 & 22.0 & & 10 \\
\hline Cs & 153.43 & 157.0 & 178.78 & 35.25 & 31.29 & 62.71 & 36.0 & 32.7 & 13.8 & 14.9 & & 10 \\
\hline $\mathrm{Be}$ & 55.94 & 50.7 & 45.56 & 17.23 & 19.19 & 31.47 & - & 11.7 & & & & 54.9 \\
\hline $\mathrm{Mg}$ & 75.71 & 44.9 & & 27.02 & 29.13 & 59.08 & 26.0 & 23.7 & & & & 17.3 \\
\hline $\mathrm{Ca}$ & 61.75 & 56.4 & & 32.61 & 35.12 & 52.45 & 33.0 & 26.4 & & & 64 & 16 \\
\hline $\mathrm{Sr}$ & 36.08 & 26.1 & & 87.12 & 76.39 & 102.63 & 85.0 & 61.1 & & & 82 & 7 \\
\hline $\mathrm{Ba}$ & 9.94 & 7.8 & & 307.23 & 300.91 & 449.22 & 306.0 & 308.0 & & & 290 & 15 \\
\hline $\mathrm{Zn}$ & 22.83 & 24.8 & & 37.83 & 38.74 & 53.04 & 37.0 & 35.6 & & & & 37 \\
\hline $\mathrm{Cd}$ & 44.25 & 34.6 & & 37.20 & 34.65 & 70.12 & 34.0 & 30.4 & & & & 23 \\
\hline $\mathrm{Hg}$ & 12.26 & 13.5 & & 91.12 & 88.93 & 90.93 & 91.0 & 102.0 & & & & 30 \\
\hline $\mathrm{Al}$ & 37.89 & 30.8 & 29.10 & 21.65 & 25.58 & 34.12 & 24.0 & 20.8 & & & & 27 \\
\hline $\mathrm{Ga}$ & 28.12 & 32.3 & & 29.63 & 26.49 & 81.18 & 26.0 & 21.6 & & & & 23 \\
\hline In & 29.51 & 30.5 & & 31.94 & 30.65 & 40.75 & 33.0 & 26.0 & & & 35.6 & 24 \\
\hline $\mathrm{Pb}$ & 100.55 & $\begin{array}{l}96.8 \\
{[10]}\end{array}$ & & 90.59 & 94.57 & 135.24 & 95.0 & $\begin{array}{l}68.9 \\
{[10]}\end{array}$ & & & 121 & 64 \\
\hline
\end{tabular}

TABLE II. Electrical resistivity of some simple and non-simple liquid metals 
It is strongly emphasis previously by Esposito et al [19] that the Ziman's formula describes electrical transport correctly for simple liquid metals while self-consistent results of resistivity are over estimates if mean free paths are comparable or smaller than an interatomic distances, which happens in the $\mathrm{d}$ and f-shell metals. In the present investigations we have calculated the electrical resistivity of simple and non-simple liquid metals using Ziman's formula, self-consistent approach and t-matrix formula have been reported. The present investigations confirm that the self consistent approach are the better choice for the resistivity calculations of simple and non-simple liquid metals.

\section{References}

[1] R. Evans, D. A. Greenwood, P. Lloyd, Phys. Letters A 35, 57 (1971).

[2] O. Dreierach, R. Evans, H. J. Guntherodt, and H. U. Kunzi, J. Phys. F 2, 709 (1972).

[3] H. B. Singh, A. Holz, Phys. Rev. A24, 1108 (1983).

[4] J. K. Percus, G. J. Yevick, Phys. Rev. 110, 1 (1958).

[5] J. K. Baria, A. R. Jani, Physica B 328, 317 (2003).

[6] J. K. Baria, A. R. Jani, Pramana J. of Phys. 60, 1235 (2003).
[7] J. S. Mc Caskili, N. H. March, Phys. Chem. Liquids 12, 1 (1982).

[8] A. Ferraz, N. H. March, Phys. Chem. Liquids 8, 271 (1979).

[9] J. Laakkonen, R. M. Nieminen, J. Phys. F 13, 2265 (1983).

[10] T. M. K. Kahjil, M. Tomak, Phys. Stat. Sol (b) 134, 321 (1986).

[11] T. E. Faber, Theory of liquid metals, Cambridge University Press, Cambridge, (1972), P-326.

[12] L. I. Yastrebov and A. A. Katsnelson, Foundation of one electron theory of solids, Mir Publishers, Moscow, (1987), P-42.

[13] R. Evans, J. Phys. C (Met. Phys. Suppl.) 2, 137 (1970).

[14] R. Evans, B. L. Gyorffy, N. Szabo, and J. M. Ziman, Properties of Liquid metals, Ed. By S. Takenohi, (1973).

[15] F. Daver, T. M. A. Khajil, and M. Tomak, Phys. Stat. Sol. (b) 138, 373 (1986).

[16] W. Geertsma, D. Gonzalez, and L. H. Gonzalez, Braz. J. Phys. 33, 406 (2003).

[17] C. R. Leavens, A. H. Macdonald, R. Taylor, A. Ferraz, and N. H. March, Phys. Chem. Liq. 11, 115 (1981).

[18] Y. Waseda, The structure of non-crystalline materials liquid and amourphous solids. Mc Graw-Hill, (1980).

[19] E. Esposito, H. Ehrenreich, and C. D. Gelatt Jr., Phys. Rev. B 18, 3913 (1978). 